\title{
Mechanical Properties Enhancement of High Reliability Metallic Materials by Laser Shock Processing
}

\author{
J.L. Ocaña, M. Morales, J.A. Porro, D. Iordachescu, M. Díaz, \\ L. Ruiz de Lara, C. Correa
}

Keywords: Laser shock processing, residual stresses, surface properties, roughness, hardness, wear, mechanical resistance, fatigue life.

\begin{abstract}
Laser shock processing (LSP) is being increasingly applied as an effective technology for the improvement of metallic materials surface properties in different types of components as a means of enhancement of their corrosion and fatigue life behavior. As reported in previous contributions by the authors, a main effect resulting from the application of the LSP technique consists on the generation of relatively deep compression residual stresses field into metallic alloy pieces allowing an improved mechanical behaviour, explicitly the life improvement of the treated specimens against wear, crack growth and stress corrosion cracking. Additional results accomplished by the authors in the line of practical development of the LSP technique at an experimental level (aiming its integral assessment from an interrelated theoretical and experimental point of view) are presented in this paper. Concretely, follow-on experimental results on the residual stress profiles and associated surface properties modification successfully reached in typical materials (especially $\mathrm{Al}$ and $\mathrm{Ti}$ alloys) under different LSP irradiation conditions are presented along with a practical correlated analysis on the protective character of the residual stress profiles obtained under different irradiation strategies and the evaluation of the corresponding induced properties as material specific volume reduction at the surface, microhardness and wear resistance. Additional remarks on the improved character of the LSP technique over the traditional "shot peening" technique in what concerns depth of induced compressive residual stresses fields are also made through the paper.
\end{abstract}

\section{Introduction}

Laser shock processing (LSP) is being increasingly applied as an effective technology for the improvement of metallic materials surface properties in different types of components as a means of enhancement of their corrosion and fatigue life behavior. Specially wear resistance, stress corrosion cracking susceptibility and crack propagation rate seem to be material properties specially improved by LSP treatments [1-4].

Although the technique was initially developed for the improvement of the fatigue cracking resistance of materials used in the aeronautic applications, materials such as Aluminum and Titanium alloys and different types of stainless steel are being extensively investigated in the frame of different areas of application, especially the aerospatial sector itself but also the nuclear, automotive and biomedical sectors on the basis of the commercial availability of new powerful laser sources able to provide intensities exceeding the $\mathrm{GW} / \mathrm{cm}^{2}$ level [5-9].

In this paper, experimental results on the residual stress profiles created in $\mathrm{Al}$ and $\mathrm{Ti}$ alloys under different irradiation conditions are presented along with a practical correlated analysis on the protective character of the residual stress profiles obtained under different irradiation strategies and. the evaluation of the corresponding induced properties as specific volume reduction at the surface, microhardness and wear resistance. 
The first type of results are of special interest from the point of view of assessing the mechanical resistance of LSP treated components (especially those designed for high fatigue life under cyclic load, while the latter are considered to be especially useful from the point of view of surface integrity preservation against corrosion processes [10] or surface modification purposes in certain types of high specificity applications (see, i.e, ref [11]).

\section{Experimental setup}

The practical irradiation system used for the experiments reported in this paper is essentially the same reported in previous contributions by the authors and is schematically and photographically shown in Fig. 1. Using purified water as confining medium, the test piece is fixed on a holder and is driven along $\mathrm{X}$ and $\mathrm{Y}$ directions by means of a computer controlled stage needed for the irradiation of extended areas of material following a pre-defined pulse overlapping strategy.

The laser light is then conducted to the interaction area by means of a reflecting mirror and a focusing lens. The control of the purity of the confining medium is important in order to avoid the formation of water bubbles or increasing concentration of impurities resulting from material ablation following the laser irradiation.
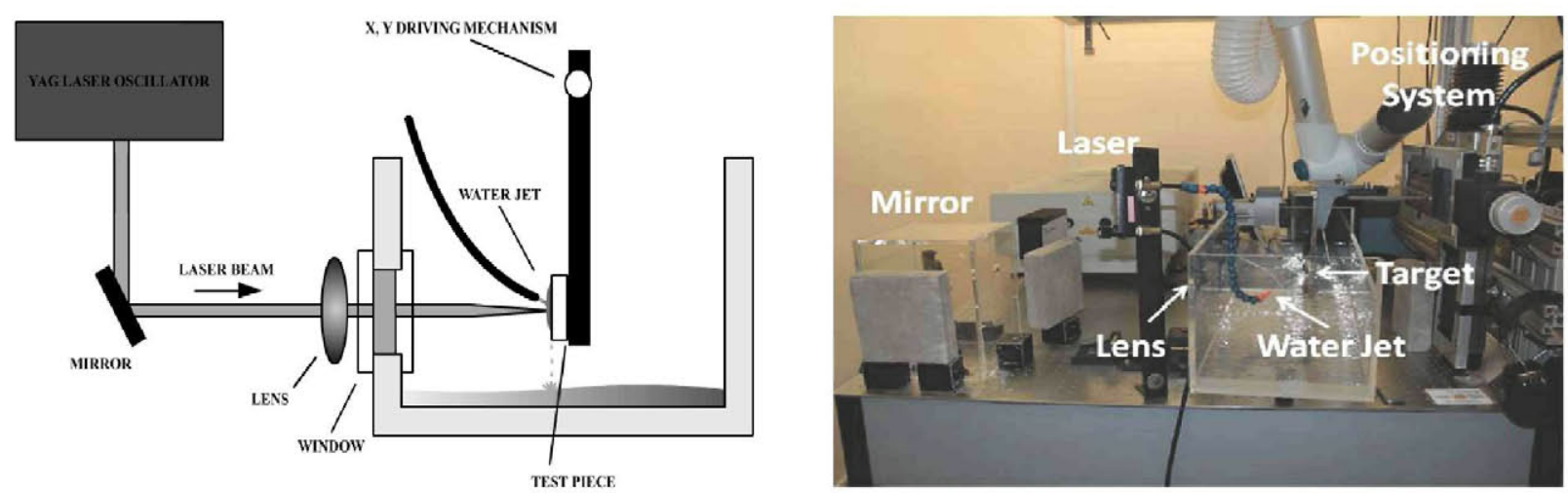

Figure 1: Schematic representation and photographic view of the LSP irradiation experimental setup used in experiments.

The LSP experiments reported in this paper were performed on Al2024-T351 and Ti6Al4V alloys at $1064 \mathrm{~nm}$ laser wavelength using a Q switched Nd:YAG laser operating at $10 \mathrm{~Hz}$ and providing $9.4 \mathrm{~ns}$ FWHM, 2.0 J pulses. A convergent lens was used to deliver the laser energy over a $1.5 \mathrm{~mm}$ spot diameter. The confining layer was provided by a water jet incident close to the laser interaction zone. No protective coatings were used in the experiments.

The irradiation geometry used for the investigations is displayed in Fig. 2 together with a photograph of the resulting aspect of the work piece after the application of the LSP treatment and subsequent residual stresses field determination by the hole drilling method.
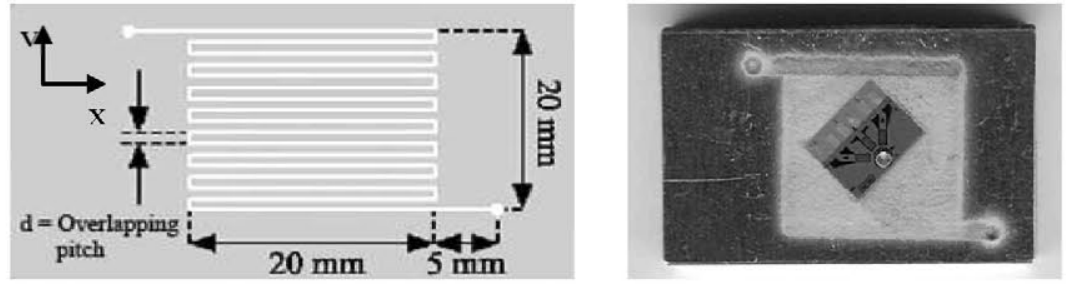

Figure 2. Schematic representation of the LSP surface sweeping strategy and photograph showing a real Al2024-T351 test piece after processing. 
In all cases, specimens of $8 \mathrm{~mm}$ thickness have been treated and tested. Values of EOD (Equivalent overlapping density (see reference [12] for definition)) of 900, 1600 and $2500 \mathrm{~cm}^{-2}$ for Al2024-T351 and of $900,2500,5000$ and $7225 \mathrm{~cm}^{-2}$ for Ti6Al4V and resulting values of ELOF (Equivalent overlapping factor, see again reference [12])) of 16, 28 and 44 for AL2024-T351 and of $16,44,88$ and 128 for Ti6Al4V were considered in the frame of the present study.

\section{Experimental results}

Under the referred experimental conditions, the effects induced by the LSP treatment on the mechanical properties of the two referred materials have been characterized. In particular, an analysis of induced residual stress fields together with its range of variability has been performed as a basis for the estimation of the practical protection degree against tensile load provided by the treatment and, additionally, associated surface modification effects, concretely microhardness and related wear resistance have been evaluated. Finally, a comparison of the LSP treatment (two different treatment intensities) applied to Ti6Al4V with respect to the results obtained in the same material by the "shot peening" technique is presented.

Residual stress distributions were determined according to the ASTM E837-01 Standard Test Method for Determining Residual Stresses by the Hole Drilling Strain Gage Method [13]. Strain gage rosettes CEA-13-062UM-120 along with a Vishay Measurements ${ }^{\circledR}$ RS-200 milling guide were used. On its side, the wear resistance tests were performed according to the ASTM G99-04 Standard Test Method for Wear Testing with a Pin on Disk Apparatus [14] on a Microtest ${ }^{\circledR}$ MT/30 system. The samples' roughness was characterized by means of a Laser Confocal Microscope Leica ${ }^{\circledR}$ ICM 1000 .

\section{Residual Stresses Fields}

Fig. 3 shows the depth profiles obtained in the two considered materials for LSP-induced Mohr maximum (i.e. minimum in absolute value) and minimum (i.e. maximum in absolute value) residual stresses for respective representative values of EOD parameter in each case.
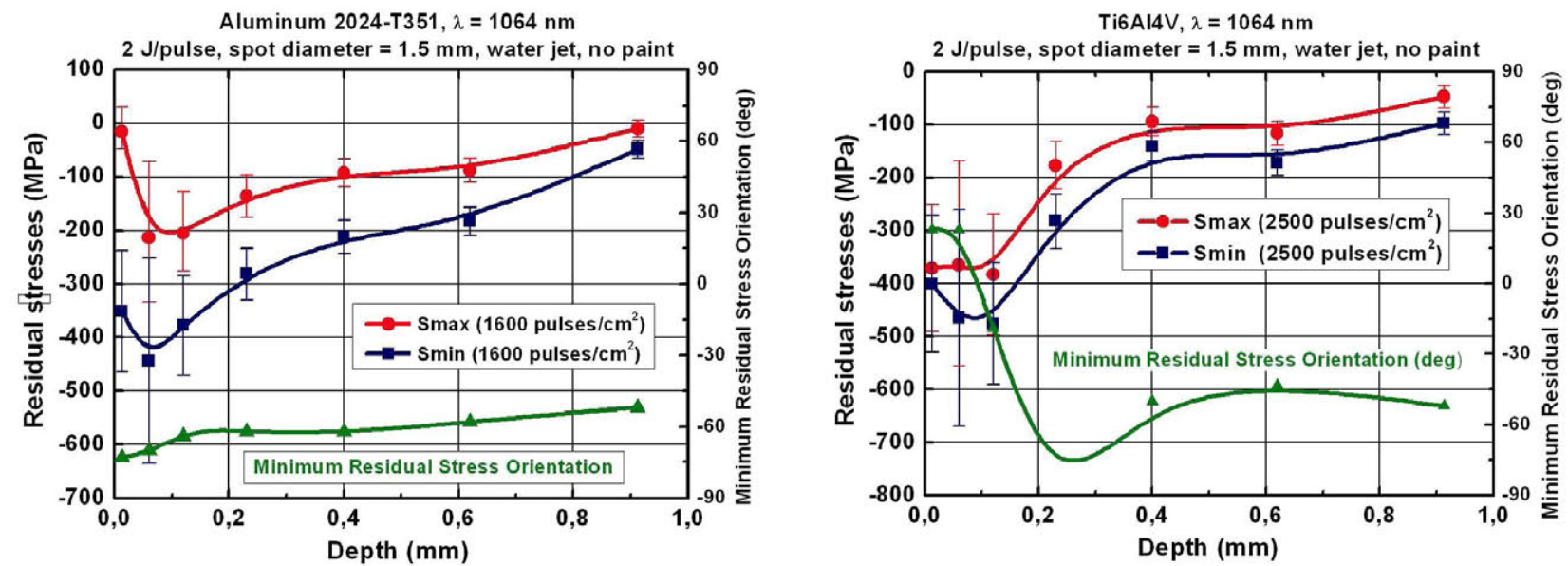

Figure 3. Depth dependence of induced Mohr maximum and minimum residual stresses induced by LSP and principal residual stress orientation in A12024-T351 and Ti6Al4V for respective representative treatment intensities (EODs).

In both cases, the effective induction of compressive residual stress fields by means of the LSP treatment is observed, so that, in practice, an effective protective field against crack aperture and propagation in depths up to near a millimetre can be assured. This effect, that can be observed with different values of the EOD parameter, is tentatively shown in figure 4, where the Mohr maxima and minima values of compressive residual stresses as a function of this parameter for both considered materials is shown. Complementarily, in figure 5, consequent theoretical regions of effective protection against different tensile load stresses are shown for both materials (in the figure corresponding to Ti6Al4V the results for A12024-T351 are also included for comparison purposes. 

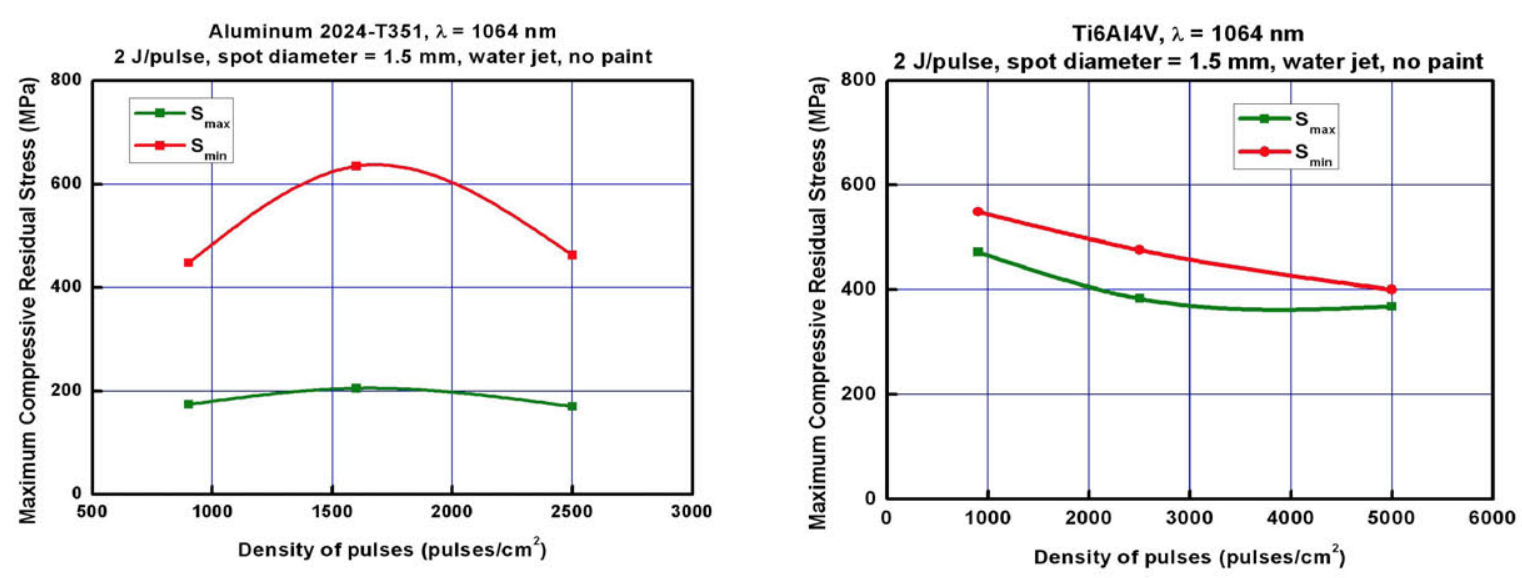

Figure 4. Variation of absolute values of compressive residual stresses Mohr maxima and minima as a function of treatment intensity (EOD parameter) in the explored ranges for Al2024-T351 and Ti6Al4V.
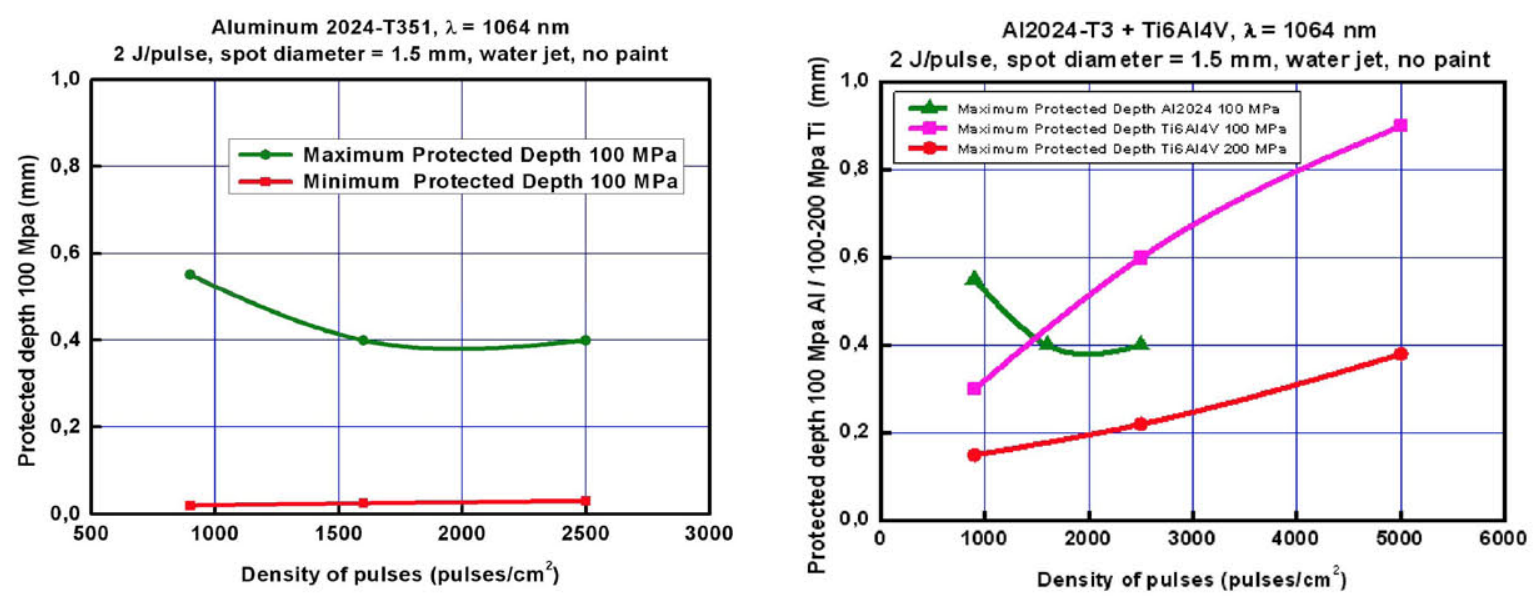

Figure 5. Theoretical regions of effective protection against different levels of applied tensile stress as deduced from the residual stresses fields induced by LSP in Al2024-T351 and Ti6Al4V as a function of treatment intensity (EOD parameter).

Taking into account the respective values of Yield Strength of each of the considered materials $\left(\mathrm{YS}_{\mathrm{Al} 2024}=290 \mathrm{MPa}, \mathrm{YS}_{\mathrm{Ti} 6 \mathrm{Al} 4 \mathrm{~V}}=880 \mathrm{MPa}\right)$, it can be observed that relatively important fractional values of pre-constraint can be reached in both materials over relatively wide depths, a characteristic feature of LSP treatments that justifies its competitive character with other related existing surface treatment technologies, namely shot peening.

From the observation of these figures, it can be deduced that, while a more or less monotonic tendency is observed in the variation with EOD of both limiting values of residual stresses in the case of Ti6Al4V (of course a direct consequence of the observed larger penetration of the protective effect observable in figure 5, in the case of A12024-T351, an optimum value of EOD seem to exist for the attainment of a maximum absolute value in both limiting values of residual stresses, with the interesting effect of the possibility of protection of larger material depths with a minimum threshold value (see again figure 5 for this case). On the other hand, in the case of Ti6Al4V, a clear positive tendency with increasing values of the EOD parameter of the maximum protected depth is observed (at least in the explored range of EODs), a fact of a great technological significance as it directly implies the possibility of selection of the intensity of the treatments as a function of the required protected working depth. In view of the relevance of all these tendencies with respect to the design of optimized protective treatments against typical ranges of tensile applied stresses, a deeper study on the possible influence of the whole set of treatment parameters needs to be accomplished. 


\section{Surface properties modification and induced hardness.}

In addition to residual stresses fields, the possible modification of the surface properties by LSP treatments is recognized as haven a decisive importance on the in-service components behaviour face to crack initiation by either mechanical or chemical (corrosion) causes (see references [9-11]). Surface roughness, for example has been identified as having a significant influence on the degradation and fatigue behaviour of several critical components, especially due to corrosion and fretting fatigue [15-16].

Although the effect can be in the contrary direction at low ELOF values, a typical consequence of the application of the LSP technique at high ELOF's is the generation on the treated surface of roughness patterns typically smoother than those of the original work-piece, a feature that has to be considered as highly favourable in comparison with the corresponding results obtained by surface treatments based on thermal processing or other mechanical treatments with comparatively lower intensity and overlapping factors, such as, typically, shot peening (see the results in this field reported by the authors in reference [12]). These results on surface roughness lowering by LSP have also a clear effect on the abrasive wear resistance of the treated specimens, although other possible surface transformation mechanisms (namely surface oxidation) finally determine the wear resistance of samples. In this case, materials able to experiment a physic-chemical transformation (carburization, nitriding, oxidation, etc.) can exhibit a differentiated behaviour concerning this property beyond the mechanical effects purely attributable to the LSP treatment.

As a clear example in this line, the comparative case of the two alloys considered in this paper (A12024-T351 and Ti6Al4V) shows the difference between the microhardness profiles obtained through LSP treatment in both materials (see figure 6).
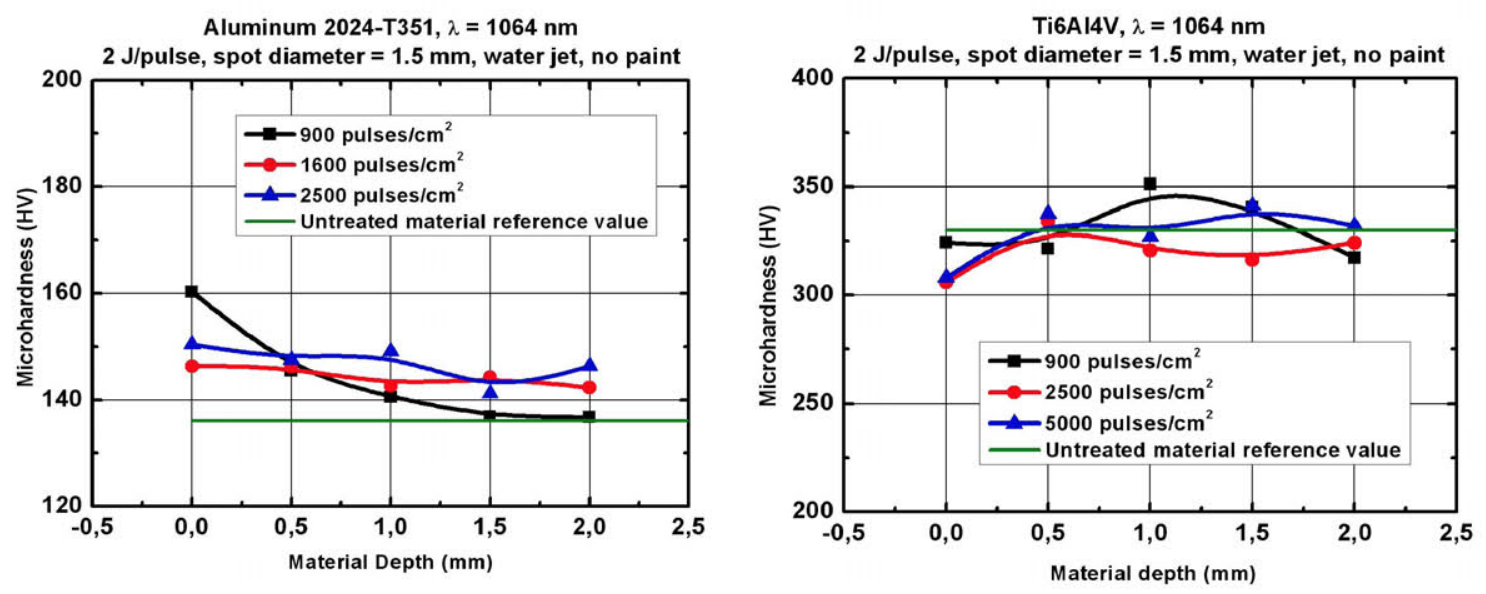

Figure 6. Microhardness profiles obtained in A12024-T351 and Ti6A14V specimens treated by LSP with different EOD's. A small hardening effect is obtained in AL2024T351, while no apparent hardening effect is observed in Ti6Al4V.

In view of the obtained results and, as suggested by the authors in a previous paper [12], after analysis of the wear behavior of both treated materials, the primary hypothesis of the appearance of the respective oxides $\left(\mathrm{Al}_{2} \mathrm{O}_{3}\right.$ and $\left.\mathrm{TiO}_{2}\right)$ as a direct consequence of the surface heating resulting from the plasma generated by the incident laser beam can be easily formulated: Although it has been shown by the authors that the effect of this plasma heating is not of a critical importance from the point of view of the residual stresses field obtained in the treated material after a certain depth [16], the effect of such heating seems to be important in what respects microstructure aspects at the surface. Precisely in view of these related effects, mostly associated with microstructure transformation, inclusion of dislocations and related specific volume reduction, etc., the subject deserves more detailed analysis, a first approach considering the effect of LSP treatment on the roughness and pitting corrosion susceptibility of AA6082 having been already published [10]. 


\section{Discussion}

In view of its primary interest for a broad range of mechanical properties susceptible to be enhanced by the LSP technique, the residual stresses depth profiles obtained in the considered materials for different treatment intensities has been presented. The observation of these results clearly leads to the consideration of the LSP technique as really effective and controllable within wide limits for the induction of engineered residual stresses fields in critical components through modification of specific mechanical and chemical material properties, especially mechanical fatigue and corrosion resistance, a fact that has been widely demonstrated by the authors [11-14].

Considering the analysis of surface microhardness and wear resistance of specimens of both materials treated by the LSP technique, the important fact has being found of the significant influence of the ambient processing conditions on the final mechanical performance of the treated surface. This matter has to be considered of a high relevance in view of its inherent difficulty to be adequately controlled and deserves a further detailed evaluation.

\section{Acknowledgements}

Work partly supported by MEC/MCINN (Spain; Projects DPI2005-09152-C02-01 and MAT200802704/MAT) UPM (Spain, Project CM CCG07-UPM/MAT-1964) and EADS-CASA (Spain).

\section{References}

[1] B.P. Fairand et al. J. Appl. Phys., 43, 3893-3896 (1997).

[2] L.C. Yang: J. Appl. Phys., 45, 2601-2607 (1974).

[3] B.P. Fairand, A.H. Clauer: J. Appl. Phys., 50, 1497-1502 (1979).

[4] Y. Sano et al.: "Underwater Laser Processing to Improve Residual Stress on Metal Surface". In: Proceedings of the 6th International Welding Symposium of Japan Welding Society, Nagoya, Japan, 501-506 (1996).

[5] C.B. Dane: "High Power Laser for Peening of Metals Enabling Production Technology". In: Lawrence Livermore National Laboratory, Report UCRL-JC-131104-Rev 1 (1998).

[6] Y. Sano et al.: Nucl. Inst. Meth. Phys. Res. B, 121, 432-436 (1997).

[7] Y.B. Guo, R. Caslaru: J. Mat. Proc. Technol. 211, 729-736 (2011).

[8] M. Rozmus-Górnikowska: Acta Physica Polonica A, 117, 808-811 (2010).

[9] S.R. Mannava et al.: Int. J. Struct. Integ. 2 , 101-113 (2011).

[10] U. Trdan et al.: Int. J. Struct. Integr., 2, $9-21$ (2011)

[11] S. Barriuso et al.: "Roughening of metallic biomaterials by abrasiveless waterjet peening: Characterization and viability“. To appear in Wear (2011). doi:10.1016/j. wear.2011.01.024

[12] J.L. Ocaña et al.: Materials Science Forum, 638-642, 2446-2451 (2010).

[13] ASTM International: "E837-01 Standard Test Method for Determining Residual Stresses by the Hole-Drilling Strain Gage Method". v.03.01. (2002)

[14] ASTM International: "ASTM G 99-04 Standard Test Method for Wear Testing with a Pin on Disk Apparatus" (2004).

[15] T. Nicholas: Int. J. Fatigue, 21, S221-S231 (1999).

[16] A.L. Hutson, T. Nicholas: Tribol. Int. 36, 133-143 (2003).

[17] J.L. Ocaña et al.: Mat. Sci. Forum Vols. 539-543, 1116-1121 (2007).

[18] C. Rubio-González et al.: Mat. Sci. Eng. A, 386, 291-295 (2004).

[19] J.L. Ocaña et al.: "Laser Shock Processing as a Method for Surface Properties Modification of Metallic Materials". In: Shot Peening and other Mechanical Surface Treatments. V. Schulze, A. Niku-Lari, Eds. I.I.T.T. Paris, 466-471 (2005).

[20] U. Sánchez-Santana et al.: Wear, 260, 847-854 (2006).

[21] C. Rubio-González et al.: Appl. Surf. Sci., 252, 6201-6205 (2006). 